\title{
Unusual case of successful treatment of a ruptured thoracoabdominal aneurysm in elderly woman
}

\author{
Grzegorz Oszkinis', Bartlomiej Perek², Anna Perek', Malgorzata Ladzinska², Lukasz Kruszyna' \\ 'Department of General and Vascular Surgery, Poznan University of Medical Sciences, Poland \\ ${ }^{2}$ Department of Cardiac Surgery and Transplantology, Poznan University of Medical Sciences, Poland \\ ${ }^{3}$ Department of Anesthesiology and Intensive Therapy, Poznan University of Medical Sciences, Poland
}

\begin{abstract}
We describe a case of a 76-year-old woman who underwent emergent surgery for ruptured thoracoabdominal aneurysm with the use of partial left heart bypass. Dacron prosthesis was implanted into the thoracic and abdominal aorta (up to its bifurcation), and four visceral arteries were reimplanted into the prosthesis. The procedure of implanting the prosthesis into the thoracic part of the descending aorta was supported by left heart bypass. The patient's postoperative course was complicated by spinal cord ischaemia-induced lower extremities paraparesis. On the $26^{\text {th }}$ postoperative day, she was referred to the regional neurological rehabilitation centre.
\end{abstract}

Key words: ruptured thoracoabdominal aneurysm, surgery, partial cardiac bypass, complications, outcomes

Acta Angiol 2015; 21, 4: 129-131

\section{Introduction}

An enormous progress made in endovascular technology has enabled successful treatment of complex pathologies of thoracoabdominal aorta with stent grafts [I]. However, manufacturing customized grafts (commercially available are usually unfit) lasts a few weeks. Thus, patients with ruptured thoracoabdominal aortic aneurysms (TAAAs) are either considered unfit for any intervention or undergo extremely high-risk surgical procedures.

This report describes the case of a 76-year-old woman who underwent a successful surgery for thoracoabdominal aortic rupture conducted with the use of partial left heart bypass.

\section{Case study}

A 76-year-old woman was admitted to the Department of General and Vascular Surgery with a symptomatic thoracoabdominal aneurysm. Although this diagnosis had been established 3 years earlier (Fig. IA), she was treated medically due to the relatively minor symptoms and the high risk of conventional surgical approach (age, renal failure, obesity, chronic obstructive pulmonary disease, diabetes treated with insulin). Moreover, in the consecutive control computed tomographic angiographies (CTA) performed once a year the diameter of thoracic and abdominal aorta was stable. Considering the progress in intravascular techniques over the last few years and our team experience in treatment with stent grafts, this time elective implantation of a customized fenestrated stent graft was selected. After necessary measurements were taken from CTA, appropriate stent graft was ordered. Unfortunately, on the day of the discharge, acute abdominal pain accompanied by hypovolaemic shock was observed. Following emergent CTA (Fig. IB) that revealed rapid abdominal aorta dilatation (more than $9 \mathrm{~cm}$ at the level of the left renal artery) and sign of its rupture, the patient was immediately transferred to the operating room.

Address for correspondence: Bartlomiej Perek, Department of Cardiac Surgery and Transplantology, Poznan University of Medical Sciences, Dluga I/2, 6I-848 Poznan, e-mail: bperek@yahoo.com 

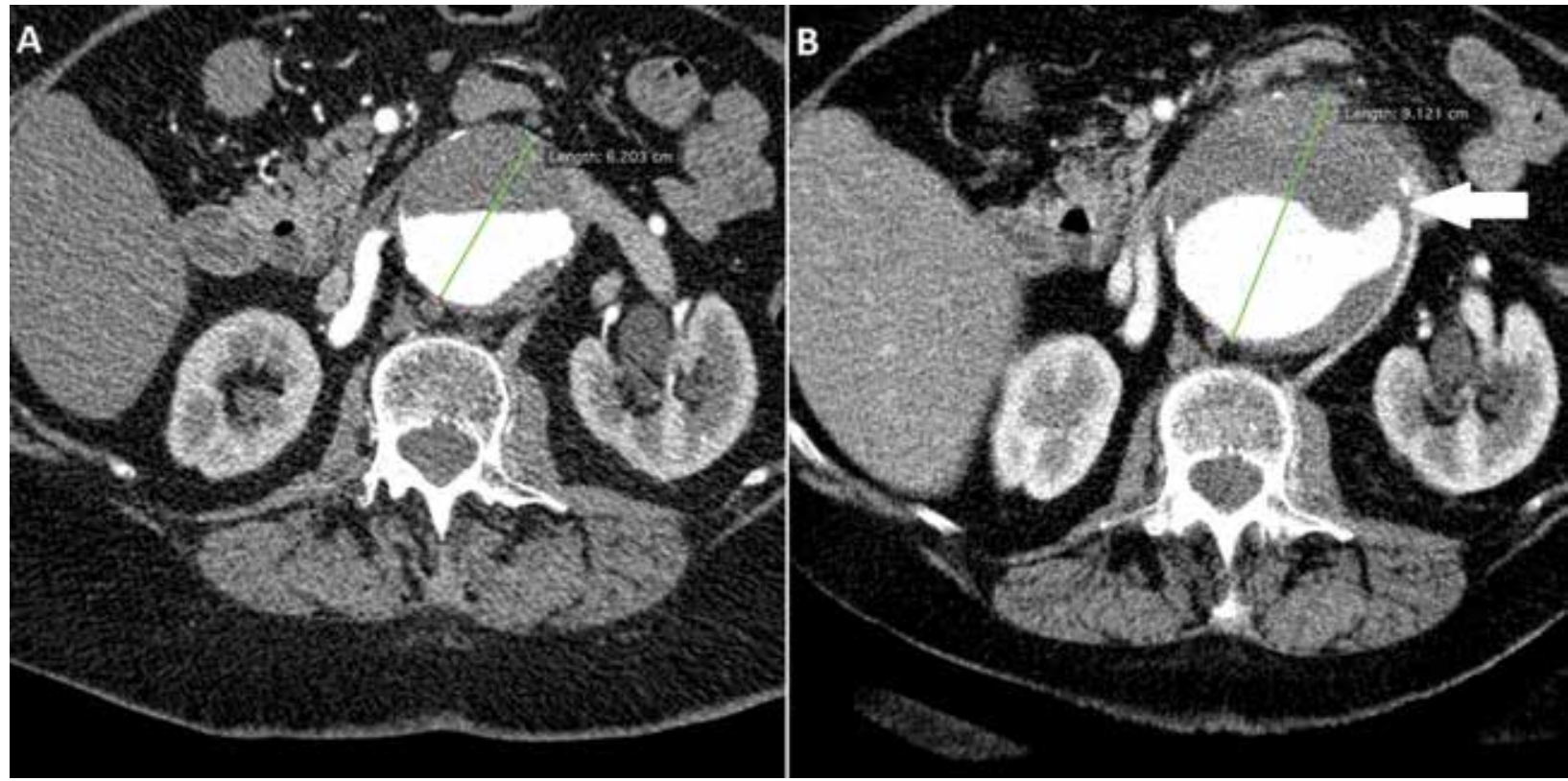

Figure IA. CTA scans of aorta at the level of renal arteries. Three years prior to surgery, CTA revealed aneurysm $(6.2 \mathrm{~cm}$ in diameter) with parietal thrombus; B. Emergent CTA (B) confirmed rupture of TAAA (arrow). Note! Diameter of abdominal aorta increased significantly to more than $9 \mathrm{~cm}$

The obligatory anaesthetic preparation included the introduction of a three-lumen central venous catheter, an endobronchial tube and a urinary catheter. To control cardiac output, an Edwards FloTrac ${ }^{\mathrm{TM}}$ sensor and a Vigileo ${ }^{\mathrm{TM}}$ monitor (Edwards Lifesciences LLC, Irvine, USA) were connected to a $20 \mathrm{G}$ radial arterial angiocatheter (Becton Dickinson, Utah, USA). We also planned to insert a cerebro-spinal fluid (CSF) drainage system but the patient's haemodynamic status deteriorated rapidly and a whole team decided to start surgery immediately. The pleural cavity was opened through the $5^{\text {th }}$ intercostal space; the thoracoabdominal incision was then extended to the abdominal wall and eventually retroperitoneal space was reached. The ruptured TAAA (Crawford type III) involved a portion of the thoracic aorta from approximately $8 \mathrm{~cm}$ above the diaphragm and the whole abdominal aorta down to the aortic bifurcation. After heparin was administered intravenously ( $150 \mathrm{IU} / \mathrm{kg}$ ), the partial extracorporeal circulation (ECC) was initiated with arterial cannula inserted into the left femoral artery and a curved venous catheter into the auricle of the left atrium. Mild hypothermia of $34.5^{\circ} \mathrm{C}$ was applied with the use of a heat exchanger integrated with ECC circuit. After the aorta was clamped proximally above the diaphragm and distally close to the bifurcation, the proximal end of a collagen-impregnated woven vascular graft (Platinum Hemashield, Maquet Cardiopulmonary, Hirrlingen, Germany) was connected to the thoracic aorta with No. 3-0 polypropylene monofilament sutures. Then proximal clamp was moved downwards, the island with the coeliac trunk, superior mesenteric artery and right renal artery was anastomosed with the vascular prosthesis with 4-0 monofilament sutures followed by reimplantation of the left renal artery. ECC was discontinued and both cannulas were removed. Eventually, the distal end of the prosthesis was connected to the end of the abdominal aorta just above its bifurcation. Following ECC completion, protamine sulphate, red cell concentrate, platelets, fresh frozen plasma and prothrombin complex (I800 IU) were transfused.

After the surgery, the patient was transferred to the Department of Intensive Therapy, where she had to be ventilated mechanically for 6 days then developed renal failure (treated successfully with continuous veno-venous haemofiltration) and excessive left pleural effusion (punctured three times). Moreover, spinal cord ischaemia diagnosed by computed tomography resulted in lower extremities paraparesis. On the $20^{\text {th }}$ day, the patient was transferred to the Surgical Department, from which, after six more days, she was referred to the neurological rehabilitation centre in good clinical condition.

\section{Discussion}

TAAA rupture is usually lethal [2]. Patients with contained ruptures, who manage to reach the hospital, have been subjected to open reconstructive surgery only by experienced surgeons. In our case, the patient 
developed symptoms suggesting TAAA rupture in the hospital. Thus, no time was lost in transferring her to our department and achieving diagnosis. However, especially in older patients, open surgery for ruptured TAAAs is associated with grave prognosis [2, 3]. Having this in mind, we initially selected this patient for endovascular treatment with a customized fenestrated stent graft. Unfortunately, due to the rupture of the TAAA she had to be operated on.

Despite the evolution of our understanding of TAAA pathophysiology and the associated operative strategies, open TAAA repair remains intrinsically related with substantial complications, of which spinal cord ischaemia and renal failure are the most devastating [2]. Several techniques were proposed to decrease the risk of malperfusion, including surgical manoeuvre of two repositioning clamps, systemic hypothermia, cerebrospinal fluid drainage, the irrigation of the epidural space with cold solutions or visceral hybrid procedures [2-5]. In our case, employing a two-clamp technique was, unfortunately, prevented by the rupture of the aneurysm and a huge haematoma around the aorta in the retroperitoneal space. We were forced to place distal clamp near the aortic bifurcation. Because of the emergency of the surgery and the fact that open surgery for TAAA is not a routine procedure, we were not able to insert any special system for draining and/or irrigating the epidural space. Moreover, we did not have stent graft of appropriate diameter. As a protection strategy, we employed a technique of left heart bypass (LHB) with mild hypothermia and distal perfusion through a femoral cannula. There are conflicting reports regarding the efficiency of this strategy [6, 7]. Schepens et al. found that the use of LHB reduced not only the rates of hospital mortality but also paraplegia/paraparesis and postoperative renal failure requiring dialysis [6]. They also revealed that the rupture of TAAA increased the risk of paraplegia or paraparesis (odds ratio 3.2). Contrary to the aforementioned study, Coselli et al. reported no favourable impact of LHB on the incidence of spinal cord injury [7]. In our opinion, the employment of LHB enabled meticulous control of body temperature throughout long-lasting procedure and blood re-infusion from the operating field. The continuous perfusion of peripheral circulation inhibited any profound ischaemia of the lower extremities. Eventually, only slight metabolic acidosis and transient mild haemodynamic instability were noted during the reperfusion phase. However in our case, LHB and hypothermia were not enough to prevent ischaemic injury to the spinal cord.
We are aware of a fact that introduction of CSF drainage system could have improved perfusion of spinal cord. Although it is not efficient in all subjects and may result in some neurological and haemorrhagic complications it is commonly used during open surgeries for TAAA [8, 9]. However, in our particular case rapid clinical and haemodynamic deterioration in the operating theatre made its application impossible.

In summary, LHB and mild controlled hypothermia used during open surgical repair for ruptured TAAA in elderly woman were essential in ensuring the survival of the patient and facilitating stable circulation during the surgery.

\section{References}

I. Tsilimparis N, Perez S, Dayama A, Ricotta JJ 2nd (2013) Endovascular repair with fenestrated-branched stent grafts improves 30-day outcomes for complex aortic aneurysms compared with open repair. Ann Vasc Surg; 27: 267-273.

2. von Meyenfeldt EM, Schnater JM, Reekers JA, Balm R (2009). An emergency visceral hybrid procedure for ruptured thoraco-abdominal aortic aneurysms. Eur J Vasc Endovasc Surg; 38: 162-168.

3. Kouchoukos NT, Masetti P, Rokkas CK, Murphy SF, Blackstone EH (200I) Safety and efficacy of hypothermic cardiopulmonary bypass and circulatory arrest for operations on the descending thoracic and thoracoabdominal aorta. Ann Thorac Surg; 72: 699-707.

4. Cambria RP, Davison JK, Carter C Brewster DC et al (2000) Epidural cooling for spinal cord protection during thoracoabdominal aneurysm repair: A five-year experience. J Vasc Surg; 31: 1093-1102.

5. Coselli JS, LeMaire SA, Köksoy C, Schmittling ZC, Curling PE (2002) Cerebrospinal fluid drainage reduces paraplegia after thoracoabdominal aortic aneurysm repair: results of a randomized clinical trial. J Vasc Surg; 35: 631-639.

6. Schepens MA, Vermeulen FE, Morshuis WJ et al (1997) Impact of left heart bypass on the results of thoracoabdominal aortic aneurysm repair. Ann Thorac Surg; 67: 1963-1967.

7. Coselli JS, LeMaire SA, Conklin LD, Adams GJ (2004) Left heart bypass during descending thoracic aortic aneurysm repair does not reduce the incidence of paraplegia. Ann Thorac Surg; 77: 1298-1303.

8. Ishikawa N, Omoto T, Oi M et al (2012) Acute subdural hematoma following thoracoabdominal aortic repair in a patient with Marfan syndrome: a rare complication of cerebrospinal fluid drainage. Ann Thorac Cardiovasc Surg; 18: 488-490.

9. Mehmedagic I, Resch T, Acosta S (2013) Complications to cerebrospinal fluid drainage and predictors of spinal cord ischemia in patients with aortic disease undergoing advanced endovascular therapy. Vasc Endovascular Surg; 47: 4I5-422. 